\title{
Uso de fragmentos florestais por anuros (Amphibia) de área aberta na região noroeste do Estado de São Paulo
}

\author{
Fernando Rodrigues da Silva ${ }^{1,3}$ \& Denise de Cerqueira Rossa-Feres ${ }^{2}$
}

Biota Neotropica $v 7$ (n2) - http://www.biotaneotropica.org.br/v7n2/pt/abstract?article+bn03707022007

\author{
Recebido em 30/11/06 \\ Versão Reformulada recebida em 22/03/07 \\ Publicado em 21/06/07 \\ ${ }^{1}$ Programa de Pós-Graduação em Biologia Animal, Laboratório de Ecologia Animal, \\ Departamento de Zoologia e Botânica, UNESP, Campus de São José do Rio Preto, \\ Rua Cristovão Colombo, 2265, CEP 15054-000, São Paulo, Brasil \\ ${ }^{2}$ Departamento de Zoologia e Botânica, UNESP, campus São José do Rio Preto, SP \\ e-mail:denise@ibilce.unesp.br \\ ${ }^{3}$ Autor para correspondência: Fernando Rodrigues da Silva, \\ e-mail:bigosbio@yahoo.com.br,www.ibilce.unesp.br
}

\begin{abstract}
Silva, F.R. and Rossa-Feres, D.C. The use of forest fragments by open-area anurans (Amphibia) in northwestern São Paulo State, Brazil Biota Neotrop. May/Aug 2007 vol. 7, no. 2. http://www.biotaneotropica. org.br/v7n2/pt/abstract?article+bn03707022007. ISSN 1676-0603.

Landscape arrangement plays a key role in community structure and composition. Our study was based on the hypothesis that forest fragments work as shelter areas for open-area anuran species. Four forest fragments were studied in the municipality of Icém, northwestern São Paulo State; the fragments were 1 to $4 \mathrm{~km}$ apart from each other. The anurans were sampled in the fragments by pitfalls, artificial shelters, and active search. Twelve species were registered (48\% of the species known for the region); three of these species (Eupemphix nattereri, Leptodactylus podicipinus, and Physalaemus cuvieri) had high abundance ( $\mathrm{n}>50$ individuals). In the pitfalls, 228 individuals belonging to 10 anuran species were captured. The high richness and abundance of specimens captured mean that forest fragments are important components for the anuran communities. Forest fragments in the Icém region can be used by anurans in three non-exclusive ways: i) as shelter areas during the dry season (five species registered); ii) as day shelters during the anuran reproductive season (six species registered in artificial shelters during the day, throughout the rainy season); and iii) as foraging areas (one specimen of Leptodactylus podicipinus found feeding on Blattaria in the fragment FM2). Therefore, the preservation of anuran populations of open habitats depends, to the same extent, on terrestrial habitats, such as forest fragments, and on the ponds where they reproduce.
\end{abstract}

Keywords: habitat, landscape, species richness, semideciduous forest, forest remnants.

\section{Resumo}

Silva, F.R. and Rossa-Feres, D.C. Uso de fragmentos florestais por anuros (Amphibia) de área aberta na região noroeste do Estado de São Paulo Biota Neotrop. May/Aug 2007 vol. 7, no. 2. http://www.biotaneotropica. org.br/v7n2/pt/abstract?article+bn03707022007. ISSN 1676-0603.

$\mathrm{O}$ arranjo da paisagem tem um papel fundamental na estrutura e organização de comunidades. Neste estudo, verificamos a hipótese de que fragmentos florestais funcionariam como áreas de refúgio para espécies de anuros de área aberta. Para isso, foram selecionados quatro fragmentos florestais em Icém, região noroeste do Estado de São Paulo, distanciados entre si de $1 \mathrm{a} 4 \mathrm{~km}$. A amostragem dos anuros nos fragmentos foi efetuada com armadilhas de queda (pitfalls), abrigos artificiais e busca ativa. Foram registradas 12 espécies (48\% das espécies conhecidas na região de Icém) das quais três, Eupemphix nattereri, Leptodactylus podicipinus e Physalaemus cuvieri, apresentaram alta abundância ( $\mathrm{n}>50$ exemplares). Apenas com as armadilhas de queda foram capturados 228 exemplares de 10 espécies de anuros. A elevada riqueza e a grande abundância de indivíduos capturados comprovam que os fragmentos florestais constituem parte importante do hábitat dessas espécies. Os resultados obtidos indicam que os fragmentos florestais da região estudada podem ser utilizados por anuros de três maneiras não excludentes: i) como áreas de refúgio durante a estação seca (cinco espécies foram registradas durante a estação seca), ii) como abrigos diurnos durante a estação reprodutiva (seis espécies registradas em abrigos artificiais durante o dia, ao longo da estação chuvosa); e iii) como área de forrageio (um exemplar de Leptodactylus podicipinus foi observado alimentando-se de Blattaria no fragmento FM2). Portanto, hábitats terrestres, como fragmentos florestais, são tão importantes para a conservação de populações de anuros de área aberta quanto as poças onde se reproduzem.

Palavras-chave: hábitat, ecologia da paisagem, riqueza de espécies, floresta estacional semidecidual, remanescentes florestais. 


\section{Introdução}

Estudos no âmbito de ecologia da paisagem são importantes, pois muitas espécies não estão confinadas a um único hábitat, mas movem-se entre hábitats ou vivem nas fronteiras entre dois hábitats. Para essas espécies, os tipos de hábitat que existem em uma escala regional são de importância crucial (Primack \& Rodrigues 2001). Os anfíbios anuros constituem um grupo adequado para estudos nessa escala, pois dependem de dois tipos de hábitat: aquático para reprodução e terrestre para forrageio, estivação, migração e dispersão (Stebbins \& Cohen 1995, Zug et al. 2001). Anuros são ectotérmicos, possuem pele permeável e, como consequiência, são suscetíveis às condições ambientais, que podem afetar sua distribuição e uso de hábitat (Duellman \& Trueb 1986). A temperatura elevada e a baixa umidade encontrada em áreas abertas devem ser limitantes para anfíbios, que apresentam alta taxa de perda de água por evaporação e pouca habilidade de dispersão (Rothermel \& Semlitsch 2002). Neste contexto, fragmentos florestais são hábitats relativamente menos perturbados em comparação com áreas agrícolas e urbanas, e proporcionam corredores para migração de anuros entre sítios de reprodução e áreas de refúgio, alimentação e estivação (Laan \& Verboom 1990, Knutson et al. 1999, Weyrauch \& Gubb Jr. 2004).

Apesar de muitos autores sugerirem que fragmentos florestais são importantes para anuros de áreas abertas (Laan \& Verboom 1990, Knutson et al. 1999, Weyrauch \& Gubb Jr. 2004), a taxa e o modo de utilização desses fragmentos são pouco ou nada conhecidos. A vegetação da região noroeste do estado de São Paulo, caracterizada como Floresta Estacional Semidecidual e Savana, restringe-se hoje a 4\% de sua área original (SMA/IF 2005), tendo sido substituída por pastagens, culturas diversas ou áreas urbanas (Probio 1998). Este cenário de intensa supressão da vegetação natural coloca essa região como a mais desmatada e fragmentada do estado, mas que, paradoxalmente, figura como um excelente modelo para testar in situ as influências dos remanescentes florestais sobre populações de anfíbios. No presente estudo investigamos, ao longo de um ano de amostragens com armadilhas de queda, procura ativa e abrigos artificiais, a hipótese de que remanescentes florestais seriam utilizados como área de refúgio por anuros de áreas abertas.

\section{Material e Métodos}

Este estudo foi desenvolvido em quatro fragmentos florestais no município de Icém (20`34' S e 49 $19^{\circ}$ 'W), noroeste do estado de São Paulo, entre setembro de 2004 e agosto de 2005. O clima da região é do tipo Tropical Quente e Úmido (Aw de Köppen), caracterizado por duas estações climáticas bem definidas: chuvosa, entre outubro e março, e uma pronunciada estação seca, entre abril e setembro, que recebe apenas $15 \%$ da precipitação total anual, que varia entre 1100 e $1250 \mathrm{~mm}$ ( $\pm 225 \mathrm{~mm}$ ) (Barcha \& Arid 1971). A região noroeste do estado de São Paulo se situa na bacia de drenagem do Alto Rio Paraná, que ocupa uma área de aproximadamente $900.000 \mathrm{~km}^{2}$ e é intensamente povoada, sendo considerada uma das bacias de drenagem mais impactadas da América do Sul (Castro et al. 2005).

Foram selecionados quatro fragmentos florestais em matriz de pastagem, distanciados entre si de 1 a $4 \mathrm{~km}$, com as seguintes características (Figura 1): i) fragmento de mata 1 (FM1), com 7,0 ha de área, possui um córrego que percorre todo o interior do fragmento e o solo apresenta grande quantidade de serapilheira. Está separado em uma das extremidades de outro fragmento menor (2,0 ha) por uma estrada de terra de 10 metros de largura e na outra extremidade, de outro fragmento maior (40 ha) por uma estrada de terra de 12 metros de largura; ii) fragmento de mata 2 (FM2), com 9,0 ha de área e circundado por áreas de pastagem, apresenta gramíneas (Poaceae) que invadem o interior do fragmento, e é separado de outro frag-

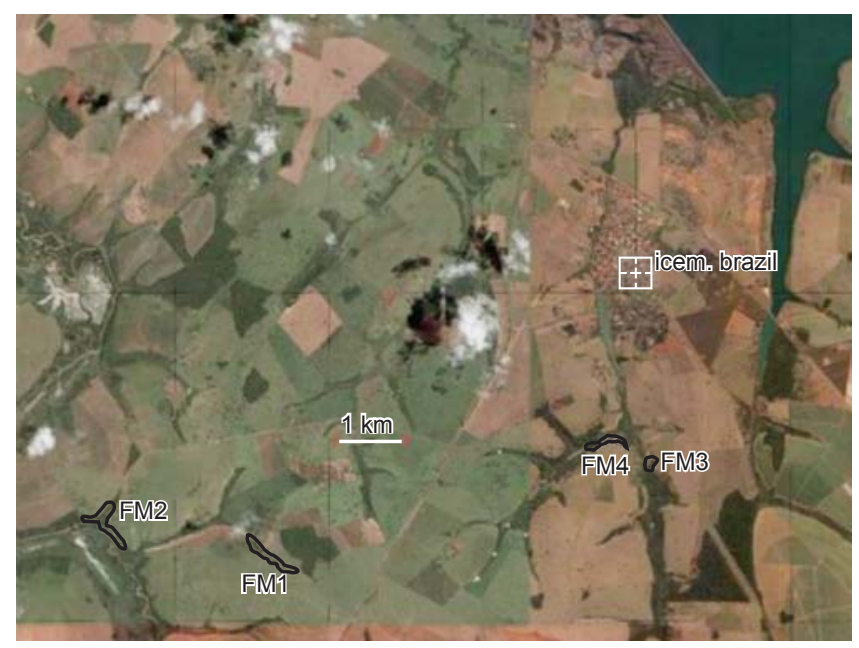

Figura 1. Fotografia aérea mostrando a área e os fragmentos florestais amostrados em Icém, SP. Fonte: Google Earth 2006.

Figure 1. Aerial photography showing the area and the forest fragments sampled in Icém, SP. Fonte: Google Earth 2006.

mento maior (16 ha) por uma área de pastagem de $60 \mathrm{~m}$ de largura; iii) fragmento de mata 3 (FM3), com 1,0 ha de área, apresenta grande quantidade de lianas e solo nu. Está separado de um fragmento maior (67 ha) por uma área de pastagem de $20 \mathrm{~m}$ de largura; e iv) fragmento de mata 4 (FM4), com 2,0 ha de área, apresenta grande quantidade de lianas, o solo seco e pedregoso, e árvores mais distanciadas entre si que nos fragmentos anteriores. Está separado de dois fragmentos maiores (22 e 6 ha) por estrada de terra com 8 m e por uma área de pastagem com $40 \mathrm{~m}$ de largura.

Foram utilizadas quatro metodologias para verificar o uso dos fragmentos florestais por anuros: i) procura visual limitada pelo tempo (PVLT sensu Campbell \& Christman 1982) que consistiu em, quinzenalmente, caminhar em velocidade lenta durante 15 minutos, procurando em todos os microambientes acessíveis por espécimes escondidos sob troncos, pedras, galhos, serapilheira etc.; e ii) abrigo artificial com pranchas de madeira (Parmelee \& Fitch 1995) de 1,2 $\times 1,0 \mathrm{~m}$, preenchidas com galhos, folhas, serapilheira e pequenas pedras. Em cada fragmento florestal foram distribuídos aleatoriamente seis abrigos artificiais, separados entre si por uma distância que variou entre 5 e $20 \mathrm{~m}$, dependendo da área do fragmento. Estes abrigos permaneceram instalados durante todo o período de desenvolvimento do projeto e foram vistoriados quinzenalmente. Os exemplares encontrados foram capturados, identificados e liberados no local.

Nos fragmentos FM1, FM2 e FM4 foram ainda aplicados outros dois métodos de amostragem, que não o foram em FM3 em razão da elevada declividade do terreno neste último fragmento: iii) abrigo artificial com canos de policloreto de vinila (PVC). Foram distribuídos aleatoriamente, em cada fragmento amostrado, quatro conjuntos com três tubos de PVC, cada um com $30 \mathrm{~cm}$ de comprimento e $6 \mathrm{~cm}$ de diâmetro. Os conjuntos de tubos permaneceram instalados durante todo o período do projeto e foram vistoriados quinzenalmente; e iv) armadilhas de queda; em cada fragmento foram demarcados dois transectos com $20 \mathrm{~m}$ de comprimento, paralelos e distantes $10 \mathrm{~m}$ entre si, contendo cada um cinco recipientes de polietileno (3 L), eqüidistantes $5 \mathrm{~m}$ e não ligados por cerca-guia. Os recipientes ficaram abertos de novembro de 2004 a agosto de 2005 e foram verificados quinzenalmente. 
Todos os indivíduos coletados foram identificados até o nível taxonômico de espécie e tiveram o sexo e a idade (juvenil ou adulto) determinados. Adultos foram sexados pelo exame da morfologia externa (presença de saco vocal e espinho nupcial em machos) e, após dissecção, pelo tamanho das gônadas. Os exemplares coletados foram depositados na Coleção Científica de Anfíbios (DZSJRP 10.093 a 10.320) do Departamento de Zoologia e Botânica da UNESP de São José do Rio Preto, SP.

\section{Resultados}

Com exceção dos abrigos artificiais com canos de policloreto de vinila (PVC), nos quais nenhuma espécie foi registrada, com os outros três métodos foram registrados anuros de 12 espécies no interior dos fragmentos florestais (Tabela 1). Na procura visual limitada pelo tempo foram registradas cinco espécies, nos abrigos artificiais com pranchas de madeira seis espécies e nas armadilhas de queda 10 espécies (Tabela 1).

As espécies mais abundantes nas armadilhas de queda foram Eupemphix nattereri, Physalaemus cuvieri e Leptodactylus podicipinus, sendo machos mais abundantes que fêmeas e juvenis (Figura 2). O número de exemplares capturados foi maior no final da estação chuvosa (fevereiro a abril) (Figura 3). O fragmento de mata 4 (FM4)

Tabela 1. Riqueza e abundância das espécies registradas no interior dos fragmentos florestais em Icém, SP, entre setembro de 2004 e agosto de 2005, determinadas pelos seguintes métodos: procura visual limitada pelo tempo, abrigos artificiais com pranchas de madeira e armadilhas de queda ("pitfall").

Table 1. Richness and abundance of the anuran species registered within forest fragments in Icém, SP, from September 2004 to August 2005, determined by visual search limited by time, artificial shelters with wooden plates, and pitfalls.

\begin{tabular}{|c|c|c|c|c|c|}
\hline Espécies & FM1 & FM2 & FM3 & FM4 & Total \\
\hline \multicolumn{6}{|l|}{ Procura Visual } \\
\hline Chaunus schneideri & - & 2 & 2 & - & 4 \\
\hline Hypsiboas raniceps & - & 2 & - & - & 2 \\
\hline Eupemphix nattereri & - & 1 & 2 & - & 3 \\
\hline Leptodactylus labyrinthicus & - & - & 1 & - & 1 \\
\hline Leptodactylus podicipinus & - & - & 1 & - & 1 \\
\hline \multicolumn{6}{|l|}{ Abrigo Artificial } \\
\hline Eupemphix nattereri & - & - & 1 & - & 1 \\
\hline Physalaemus cuvieri & 5 & 5 & 3 & - & 13 \\
\hline Leptodactylus podicipinus & 1 & 5 & 2 & - & 8 \\
\hline Leptodactylus mystacinus & 1 & - & - & - & 1 \\
\hline Leptodactylus labyrinthicus & - & - & 6 & - & 6 \\
\hline Scinax fuscovarius & - & - & 1 & - & 1 \\
\hline \multicolumn{6}{|l|}{ Armadilha de Queda } \\
\hline Eupemphix nattereri & 3 & 1 & - & 89 & 93 \\
\hline Physalaemus cuvieri & 42 & 5 & - & 12 & 59 \\
\hline Leptodactylus fuscus & - & - & - & 4 & 4 \\
\hline Leptodactylus podicipinus & 15 & 38 & - & 1 & 54 \\
\hline Leptodactylus labyrinthicus & - & - & - & 1 & 1 \\
\hline Leptodactylus mystacinus & - & - & - & 2 & 2 \\
\hline $\begin{array}{l}\text { Pseudopaludicola aff. } \\
\text { falcipes }\end{array}$ & 4 & 1 & - & - & 5 \\
\hline Chaunus schneideri & 3 & 7 & - & 1 & 11 \\
\hline Elachistocleis sp. & - & - & - & 1 & 1 \\
\hline Dermatonotus muelleri & - & 2 & - & 1 & 3 \\
\hline
\end{tabular}

apresentou a maior riqueza e abundância de anuros, com dominância de E. nattereri (79\%) (Tabela 1). Além disso, as espécies dominantes diferiram em cada fragmento: $L$. podicipinus $(70,4 \%)$ no FM2 e P. cuvieri $(62,7 \%)$ no FM1 (Tabela 1). Exemplares de E. nattereri, P. cuvieri e L. podicipinus foram capturados ao longo de todo o período de estudo, enquanto as sete espécies restantes foram registradas somente na estação chuvosa (Tabela 2).

$\mathrm{Na}$ amostragem com abrigos artificiais, a maior riqueza e abundância de anuros foram registradas no FM3 (Tabela 1). Exemplares de Leptodactylus podicipinus foram encontrados ao longo do período estudado, de Physalaemus cuvieri e de L. labyrinthicus foram encontrados durante a estação chuvosa (Tabela 3). As três espécies

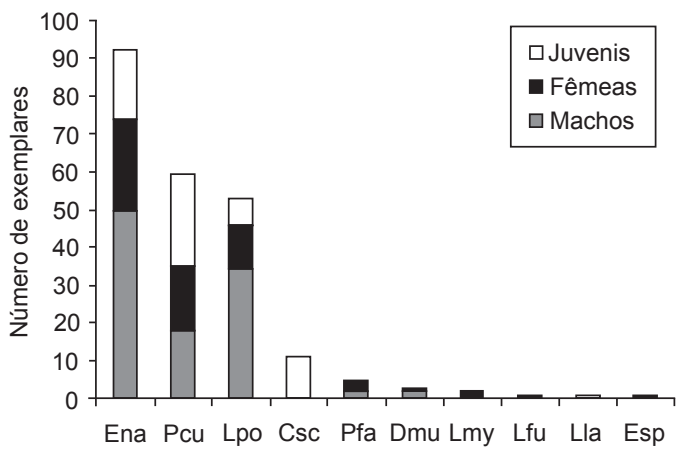

Figura 2. Total de machos, fêmeas e juvenis capturados nas armadilhas de queda (pitfall), instaladas no interior de fragmentos florestais em Icém, SP, entre novembro de 2004 e julho de 2005. Ena = Eupemphix nattereri, $\mathrm{Pcu}=$ Physalaemus cuvieri, Lpo $=$ Leptodactylus podicipinus, $\mathrm{Csc}=$ Chaunus schneideri, $\mathrm{Pfa}=$ Pseudopaludicola aff. falcipes, $\mathrm{Dmu}=$ Dermatonotus muelleri, Lmy = Leptodactylus mystacinus, Lfu = Leptodactylus fuscus, Lla $=$ Leptodactylus labyrinthicus $\mathrm{e} \mathrm{Esp} \mathrm{=} \mathrm{Elachistocleis} \mathrm{sp}$.

Figure 2. Total males, females, and juveniles captured in the pitfalls installed within the forest fragments in Icém, SP, from November 2004 to July 2005. Ena = Eupemphix nattereri, $\mathrm{Pcu}=$ Physalaemus cuvieri, Lpo $=$ Leptodactylus podicipinus, $\mathrm{Csc}=$ Chaunus schneideri, $\mathrm{Pfa}=$ Pseudopaludicola aff. falcipes, Dmu $=$ Dermatonotus muelleri, Lmy $=$ Leptodactylus mystacinus, $\mathrm{Lfu}=$ Leptodactylusfuscus, $\mathrm{Lla}=$ Leptodactylus labyrinthicus e Esp = Elachistocleis $\mathrm{sp}$.

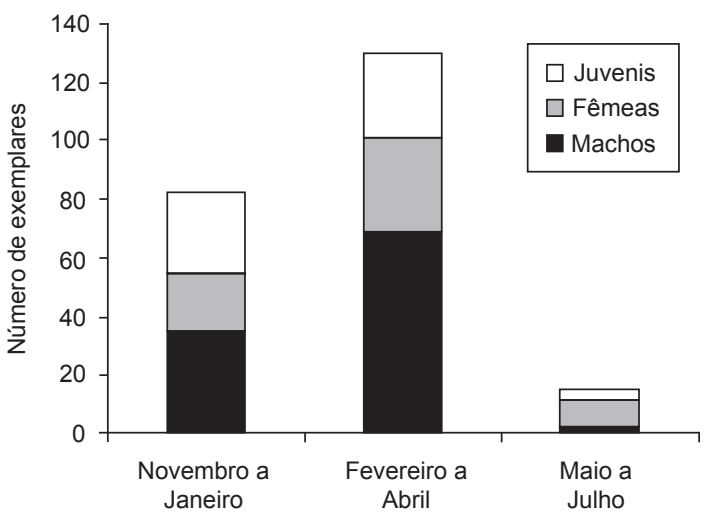

Figura 3. Ocorrência sazonal de machos, fêmeas e juvenis de todas as espécies de anuros capturados nas armadilhas de queda ("pitfall"), instaladas no interior de fragmentos florestais, em Icém, SP, entre novembro de 2004 a julho de 2005.

Figure 3. Seasonal occurrence of males, females, and juveniles of all anuran species, captured in the pitfalls installed within the forest fragments in Icém, SP, from November 2004 to July 2005. 
Tabela 2. Distribuição temporal (mensal) das espécies de anuros em armadilhas de queda, instaladas no interior de fragmentos florestais em Icém, SP, entre novembro de 2004 e agosto de 2005.

Table 2. Monthly distribution of the anuran species in the pitfalls installed within the forest fragments in Icém, SP, from November 2004 to August 2005.

\begin{tabular}{|c|c|c|c|c|c|c|c|c|c|c|}
\hline Espécies & $\mathbf{N}$ & D & $\mathbf{J}$ & $\mathbf{F}$ & $\mathbf{M}$ & $\mathbf{A}$ & $\mathbf{M}$ & $\mathbf{J}$ & $\mathbf{J}$ & $\mathbf{A}$ \\
\hline Eupemphix nattereri & 18 & 6 & 5 & 19 & 29 & 13 & 1 & - & - & - \\
\hline Physalaemus cuvieri & 11 & 10 & 3 & 8 & 12 & 3 & 7 & 2 & 3 & - \\
\hline Leptodactylus fuscus & - & - & - & - & 1 & - & - & - & - & - \\
\hline Leptodactylus podicipinus & 4 & 3 & 5 & 14 & 15 & 10 & 1 & 1 & - & - \\
\hline Leptodactylus labyrinthicus & - & 1 & - & - & - & - & - & - & - & - \\
\hline Leptodactylus mystacinus & - & - & - & 1 & - & 1 & - & - & - & - \\
\hline Pseudopaludicola aff. falcipes & 1 & 1 & - & 1 & 1 & 1 & - & - & - & - \\
\hline Chaunus schneideri & 7 & 4 & - & - & - & - & - & - & - & - \\
\hline Elachistocleis sp. & - & - & 1 & - & - & - & - & - & - & - \\
\hline Dermatonotus muelleri & - & 3 & - & - & - & - & - & - & - & - \\
\hline Riqueza & 5 & 7 & 4 & 5 & 5 & 5 & 3 & 2 & 1 & - \\
\hline
\end{tabular}

Tabela 3. Distribuição temporal (mensal) das espécies de anuros em abrigos artificiais, instalados no interior de fragmentos florestais em Icém, SP, entre setembro de 2004 e agosto de 2005.

Table 3. Monthly distribution of the anuran species in the artificial shelters installed within the forest fragments in Icém, SP, from September 2004 to August 2005.

\begin{tabular}{|c|c|c|c|c|c|c|c|c|c|c|c|c|}
\hline Espécies & $\mathbf{S}$ & $\mathbf{O}$ & $\mathbf{N}$ & $\mathbf{D}$ & $\mathbf{J}$ & $\mathbf{F}$ & $\mathbf{M}$ & $\mathbf{A}$ & $\mathbf{M}$ & $\mathbf{J}$ & $\mathbf{J}$ & $\mathbf{A}$ \\
\hline Eupemphix nattereri & - & - & - & - & - & - & - & - & - & - & - & 1 \\
\hline Physalaemus cuvieri & 1 & 4 & 1 & 3 & 3 & 1 & - & - & - & - & - & - \\
\hline Leptodactylus podicipinus & - & - & - & 2 & - & - & 1 & 2 & - & - & 2 & 1 \\
\hline Leptodactylus mystacinus & - & - & - & - & - & - & 1 & - & - & - & - & - \\
\hline Leptodactylus labyrinthicus & - & - & - & - & - & 2 & 4 & - & - & - & - & - \\
\hline Scinax fuscovarius & - & - & - & - & 1 & - & - & - & - & - & - & - \\
\hline Riqueza & 1 & 1 & 1 & 2 & 2 & 2 & 3 & 1 & - & - & 1 & 2 \\
\hline
\end{tabular}

restantes foram raras, com apenas um exemplar capturado (Tabela 3). Pelo método de procura visual limitada pelo tempo foram registrados anuros em apenas dois (FM2 e FM3) dos quatro fragmentos de mata amostrados (Tabela 1).

\section{Discussão}

O isolamento e a perda de habitat associados com a conversão de atividades humanas constituem a maior ameaça para a diversidade biológica da terra (Primack \& Rodrigues 2001, Pires et al. 2006, Watling \& Donnelly 2006). Relações negativas entre fragmentação de habitat e riqueza de espécies foram documentadas para diferentes táxons de vertebrados: mamíferos (Scott et al. 2006, Laidlaw 2006), aves (Boulinier et al. 2001, Scott et al. 2006) e lagartos (Hokit \& Branch 2003, Scott et al. 2006 ). Diversos estudos sugerem que anfíbios em paisagens alteradas são suscetíveis a fragmentação de florestas (Laan \& Verboom 1990, Fahrig et al. 1995, Gibbs 1998), agricultura (Lehtinen et al. 1999, Knutson et al. 1999), presença de estradas (Findlay \& Houlaban 1997) e isolamento de outras poças (Laan \& Verboom 1990, Vos e Stumpel 1995). Os esforços combinados desses estudos indicam que os impactos negativos do uso da terra afetam os anfíbios por reduzir a quantidade e a qualidade de poças disponíveis, habitats para o forrageio e por reduzir a conectividade ecológica da paisagem (Herrmann et al. 2005, Cushman 2006).

Das 25 espécies registradas em Icém, SP (Apêndice 1, F.R. Silva, dados não publicados), $48 \%$ (12 espécies) foram encontradas no interior dos fragmentos florestais. Tendo em vista que essas espécies são consideradas típicas de áreas abertas (sensu Duellman 1999), a riqueza registrada nos fragmentos florestais pode ser considerada alta. Apenas nas armadilhas de queda foram capturados 228 exemplares de 10 espécies, das quais três foram muito abundantes ( $n>50$ exemplares). A elevada riqueza e a grande abundância de exemplares capturados em armadilhas de pequeno volume (3 L), e sem rede direcionadora, indica que os fragmentos florestais constituem parte importante do hábitat dessas espécies.

Devido à capacidade de empoleiramento, espécies pertencentes à família Hylidae são difíceis de serem registradas com os métodos utilizados no presente estudo. Mesmo assim, duas espécies (Hypsiboas raniceps e Scinax fuscovarius) foram registradas no interior dos fragmentos florestais com as metodologias aplicadas. Além disso, em cinco ocasiões, machos de Dendropsophus minutus foram escutados vocalizando no interior dos fragmentos florestais FM1 e FM4, antes de se deslocarem para os corpos d'água (F.R. Silva, dados não publicados). Portanto, o número de espécies utilizando os fragmentos florestais pode ser maior que o registrado com os métodos empregados.

Diversos estudos indicam que os anuros podem utilizar fragmentos florestais como área de refúgio (Knutson et al. 1999), forrageio, hibernação e migração (Stebbins \& Cohen 1995, Gibbs 1998, Marsh \& Trenham 2001, Weyrauch \& Gubb Jr. 2004). A captura de anuros adultos durante a estação seca reforça a importância dos fragmentos florestais como áreas de refúgio. A diminuição no número de capturas (6,6\% do total de 228 exemplares) parece refletir a menor atividade dos anuros durante este período do ano, em que as condições de 
umidade (Barcha \& Arid 1971) e a oferta de alimento (Pinheiro et al. 2002) são menores. Várias espécies são encontradas enterradas durante a estação seca nessa região (D.C.Rossa-Feres, dados não publicados) e algumas, como Dermatonotus muelleri, entram em estivação permanecendo enterradas a mais de $50 \mathrm{~cm}$ de profundidade por até sete meses (Nomura 2003). Segundo Moreira \& Barreto (1996), várias espécies como Scinax fuscovarius e Physalaemus cuvieri, registradas no presente estudo, já foram encontradas abrigadas dentro de cupinzeiros no cerrado durante este período.

Muitos imagos foram capturados nas armadilhas de queda no final da estação chuvosa (fevereiro a abril), indicando que os juvenis recém-metamorfoseados deslocam-se para os fragmentos florestais em busca de refúgio e/ou alimento pois, devido a alta taxa de dessecação apresentada por esses organismos (Rothermel \& Semlitsch 2002), a severa estação seca típica da região noroeste do estado (Barcha \& Arid 1971), muito provavelmente restringe os deslocamentos e dispersão de anuros aos meses da estação chuvosa. Resultados semelhantes foram obtidos por Gibbs (1998), que registrou alta taxa de captura de juvenis recém metamorfoseados deixando áreas abertas em direção a fragmentos florestais em Connecticut, Estados Unidos, e por Demaynadier \& Hunter (1999), que demonstraram que juvenis de Lithobates sylvaticus migram para florestas imediatamente após a metamorfose. A importância dos fragmentos como área de forrageio é aventada a partir da observação anedótica de um exemplar de Leptodactylus podicipinus alimentando-se de Blattaria em um dos refúgios artificiais instalado no fragmento FM2. Blattaria foi encontrada na dieta de exemplares dessa mesma espécie no Pantanal (Rodrigues et al. 2004) e em Icém, SP (F.R. Silva, dados não publicados). Goehring et al. (2002) em um estudo da distribuição de artrópodes em florestas e áreas de cultivo na Costa Rica, registraram que insetos de quatro Ordens (Blattaria, Coleptera, Díptera e Orthoptera) foram mais abundantes em fragmentos de mata que em áreas de cultivo. Assim, anuros que se alimentam de insetos pertencentes a esses grupos taxonômicos podem utilizar os fragmentos como áreas de forrageio.

Sete espécies (64\%) foram capturadas nas armadilhas de queda apenas na estação chuvosa, reforçando a idéia de Hazell et al. (2001) e Guerry \& Hunter Jr. (2002) de que a ocorrência de algumas espécies de áreas abertas está associada com a presença de fragmentos florestais próximos a poças para reprodução. Segundo F.R. Silva (dados não publicados), Dendropsophus minutus, D. nanus, Eupemphix nattereri, Leptodactylus fuscus, L. podicipinus, Physalaemus cuvieri, Scinax fuscovarius e $S$. similis foram mais abundantes em corpos d'água próximos a fragmentos florestais do que em corpos d'água distantes desses fragmentos. Assim, os fragmentos florestais podem funcionar como corredores para deslocamento de anuros entre hábitats de reprodução e áreas onde realizam outras atividades como alimentação, hibernação e estivação (Laan \& Verboom 1990, Knutson et al. 1999, Weyrauch \& Gubb Jr. 2004), uma vez que estradas e outros ambientes hostis devem provavelmente constituir barreiras para seu deslocamento (Gibbs 1998).

O delineamento amostral não permitiu verificar a hipótese dos fragmentos serem áreas importantes para dispersão, como proposto por vários autores (e.g. Stebbins \& Cohen 1995, Gibbs 1998), mas os resultados obtidos indicam que os fragmentos florestais da região estudada podem ser utilizados por anuros de três maneiras não excludentes: i) como áreas de refúgio durante a estação seca (cinco espécies registradas durante a estação seca), ii) como abrigos diurnos durante a estação reprodutiva (seis espécies registradas em abrigos artificiais durante o dia, ao longo da estação chuvosa); e iii) como área de forrageio (um exemplar de Leptodactylus podicipinus alimentandose de Blattaria no fragmento FM2). Portanto, hábitats terrestres, como fragmentos florestais, são tão importantes para a conservação de populações de anuros de área aberta quanto as poças onde se reproduzem.

\section{Agradecimentos}

Somos gratos a Dra. Lílian Casatti, pela revisão crítica do texto e pelas importantes sugestões; a Carolina P. Candeira e Rodrigo A. Silva pelo auxílio nas atividades de campo e ao Vitor H.M. do Prado pelas sugestões. Este estudo foi financiado pela FAPESP (Biota/Fapesp proc. 01/13341-3). F.R. Silva agradece à FAPESP (Proc. 04/12223-5) pela bolsa de mestrado.

\section{Referências Bibliográficas}

BARCHA, S.F. \& ARID, F.M. 1971. Estudo da evapotranspiração na região norte-ocidental do Estado de São Paulo. Revista de Ciências da Faculdade de Ciências e Letras. 1: 94-122.

BOULINIER, T., MCCOY, K.D. \& SORCI. G. 2001. Dispersal and parasitism. In. Dispersal. (J. Clobert, E. Danchin, A. Dhondt \& J. D. Nichols, eds). Oxford University Press, Oxford, p.169-179

CAMPBELL, H.W. \& CHRISTMAN, S.P. 1982. Field techniques for herpetofaunal community analysis. In Herpetological communities: a symposium of the society for study of amphibians and reptiles and the herpetologist's league (N.J. Scott Jr,. ed.). Wildlife Research Report 13, U.S. Department of the Interior, Fish and Wildlife Service, Washington, DC.

CASTRO, R.M.C., CASATTI, L., SANTOS, H.F., VARI, R.P., MELO, A.L.A., MARTINS, L.S. F., ABREU, T.X., BENINE, R.C., GIBRAN, F.Z., RIBEIRO, A.C., BOCKMANN, F.A., CARVALHO, M., PELIÇÃO, G.Z.P., FERREIRA, K.M., STOPIGLIA, R. \& AKAMA, A. 2005. Structure and composition of the stream ichthyofauna of four tributary rivers of the upper Paraná basin, Brazil. Ichtyol. Explor. Fres. 16(3): 193-214.

CUSHMAN, S.A. 2006. Effects of habitat loss and fragmentation on amphibians: a review and prospectus. Biol. Conserv. 128(2): 231-240.

DEMAYNADIER, P.G. \& HUNTER, M.L. 1999. Forest canopy closure and juvenile emigration by pool-breeding amphibians in Maine. Journal of Wildlife Management. 63: 441-450.

DUELLMAN, W.E. \& TRUEB, L. 1986. Biology of Amphibians. McGrawHill Book Company, New York.

DUELLMAN, W.E. 1999. Distribution Patterns of Amphibians in the South America. In Patterns of Distribution of Amphibians - A Global Perspective. (W.E. Duellman ed.). Johns Hopkins University Press, Baltimore and London.

FAHRIG, L., PEDLAR, J.H., POPE, S. E., TAYLOR, P.D. \& WEGNER, J.F. 1995. Effect of road traffic on amphibian density. Biol. Conserv. 73: $177-182$.

FINDLAY, C. S. \& HOULAHAN, J. 1997. Anthropogenic correlates of species richness in Southeastern Ontario Wetlands. Conserv. Biol. 11(4): 1000-1009.

GIBBS, J.P. 1998. Amphibian movements in response to forest edges, roads, and streambeds in Southern New England. J. Wildlife Manage. 62(2): 584-589.

GOEHRING, D.M., DAILY, G.C. \& SEKERÇIOGLU, Ç.H. 2002. Distribution of ground-dwelling arthropods in tropical countryside habitats. J. Insect Conserv. 6: 83-91.

GUERRY, A.D. \& HUNTER JR. 2002. Amphibian distributions in a landscape of forest and agriculture: an examination of landscape composition and configuration. Conserv. Biol. 16(3): 745-754.

HAZELL, D., CUNNINGHAM, D.L., MACKEY, B. \& OSBORNE, W. 2001. Use of farm dams as frog habitat in an Australian agricultural landscape: factors affecting species richness and distribution. Biol. Conserv. 102: 155-169.

HERRMANN, H.L., BABBITT, K.J., BABER, M.J. \& CONGALTON, R.G. 2005. Effects of landscape characteristics on amphibian distribution in a forest-dominated landscape. Biol. Conserv. 123:139-149. 
HOKIT, D.G. \& BRANCH, L.C. 2003 Habitat patch size affects demographics of the Florida scrub lizard (Sceloporus woodi). J. Herpetol. 37(2): 257-265.

KNUTSON, M.G., SAUER, J.R., OLSEN, D.A., MOSSMAN, M.J., HEMESATH, L.M. \& LANNOO, M.J. 1999. Effects of landscape composition and wetland fragmentation on frog and toad abundance and species richness in Iowa and Wisconsin, U.S.A. Conserv. Biol. 13(6): 1437-1446.

LAAN, R. \& VERBOOM, B. 1990. Effects of pool size and isolation on amphibian communities. Biol. Conserv. 54: 251-262.

LAIDLAW, R.K. 2006. Effects of habitat disturbance and protected areas on mammals of Peninsular Malaysia. Conserv. Biol. 14(6): 1639-1648.

LEHTINEN, R. M., GALATOWITSCH, M. J. \& TESTER, R. 1999. Consequences of habitat loss and fragmentation for wetland amphibian assemblages. Wetlands 19:1-12.

MARSH, D.M. \& TRENHAM, P.C. 2001. Metapopulation dynamics and Amphibian conservation. Conserv. Biol. 15(1): 40-49.

MOREIRA, G. \& BARRETO, L. 1996. Alimentação e variação sazonal na freqüência de captura de anuros em duas localidades do Brasil Central. Rev. Bras. Zool. 13: 313-320.

NOMURA, F. 2003. Ecologia reprodutiva e comportamento de forrageio e escavação de Dermatonotus muelleri (Boettger, 1885) (Anura, Microhylidae).Dissertação de mestrado, Universidade Estadual Paulista, São José do Rio Preto.

PARMELLE, J.R. \& FITCH, H.S. 1995. An experiment with artificial shelters for snakes: effects of material, age, and surface preparation. Herpetological Natural History. 3:187-191.

PINHEIRO, F., DINIZ, I.R., COELHO, D. \& BANDEIRA, M.P.S. 2002. Seasonal pattern of insect abundance in the Brazilian cerrado. Austral Ecology. 27:132-136.

PIRES, A.S., FERNANDEZ, F.A.S. \& BARROS, C.S. 2006. Vivendo em um mundo em pedaços: Efeitos da fragmentação florestal sobre comunidades e populações de animais. In. Biologia da conservação: essências (C.F.D. Rocha, H.G. Bergallo, M.Van-Sluys \& M.A.S. Alves, eds) RiMa Editora, São Carlos, p.231-260
PRIMACK, R.B. \& RODRIGUES, E. 2001. Biologia da conservação. Gráfica e editora Midiograf, Londrina.

PROBIO 1998. Áreas de domínio do cerrado no Estado de São Paulo. Imprensa Oficial, Secretaria do Meio Ambiente de São Paulo, São Paulo.

RODRIGUES, D.J., UETANABARO, M. \& PRADO, C.P.A. 2004. Seasonal and ontogenetic variation in diet composition of Leptodactylus podicipinus (Anura, Leptodactylidae) in the southern Pantanal, Brazil. Rev. Esp. Herp. 18:19-28.

ROTHERMEL, B.B., \& SEMLITSCH, R.D. 2002. An experimental investigation of landscape resistance of forest versus old-field habitats to emigrating juvenile amphibians. Conserv. Biol. 16:1324-1332.

SCOTT, D.M., BROWN, D., MAHOOD, S., DENTON, B., SILBURN, A. \& RAKOTONDRAPARANY, F. 2006. The impacts of forest clearance on lizards, small mammal and bird communities in the arid spiny forest, southern Madagascar. Biol. Conserv. 127:72-87.

SMA/IF (Secretaria do Meio Ambiente / Instituto Florestal). 2005. Inventário florestal da vegetação natural do Estado de São Paulo. Imprensa Oficial do Estado de São Paulo, São Paulo.

STEBBINS, R.C. \& COHEN, N.W. 1995. A Natural History of Amphibians. Princeton University Press, New Jersey.

VOS, C. C. \& A. H. P. STUMPEL. 1995. Comparison of habitat-isolation parameters in relation to fragmented distribution patterns in the tree frog (Hyla arborea). Landscape Ecol. 11: 203-214.

WATLING, J.I. \& DONNELLY, M.A. 2006. Fragments as Islands: a synthesis of faunal responses to habitat patchiness. Conserv. Biol. 20(4): 1016-1025.

WEYRAUCH, S.L. \& GRUBB JR. 2004. Patch and landscape characteristics associated with the distribution of woodland amphibians in a agricultural fragmented landscape: an information-theorectic approach. Biol. Conserv. 115: 443-450.

ZUG, G.R., VITT, L.J. \& CALDWELL, J.P. 2001. Herpetology: an introductory biology of amphibians and reptiles. 2 ed. Academic Press, San Diego. 


\section{Apêndice 1.}

Lista das espécies de anuros registradas entre setembro de 2004 e agosto de 2005, em Icém, SP. Fase da vida: $\mathrm{A}=$ adulto e $\mathrm{G}=\mathrm{Girino}$.

\section{Appendix 1.}

Species list of anuran registered from September 2004 to August 2005, in Icém, SP. Phase of the life: A = adult and G = tadpole

\begin{tabular}{|c|c|c|}
\hline Família & Espécie & Fase da Vida \\
\hline Bufonidae & Chaunus schneideri (Werner, 1894) & $\mathrm{A} / \mathrm{G}$ \\
\hline \multirow[t]{10}{*}{ Hylidae } & Dendropsophus elianeae (Napoli \& Caramaschi, 2000) & $\mathrm{A} / \mathrm{G}$ \\
\hline & Dendropsophus minutus (Peters, 1872) & $\mathrm{A} / \mathrm{G}$ \\
\hline & Dendropsophus nanus (Boulenger, 1889) & $\mathrm{A} / \mathrm{G}$ \\
\hline & Hypsiboas albopunctatus (Spix, 1824) & $\mathrm{A} / \mathrm{G}$ \\
\hline & Hypsiboas raniceps Cope, 1862 & A \\
\hline & Pseudis paradoxa (Linnaeus, 1758) & A \\
\hline & Scinax fuscomarginatus (Lutz, 1925) & A \\
\hline & Scinax fuscovarius (Lutz, 1925) & $\mathrm{A} / \mathrm{G}$ \\
\hline & Scinax similis (Cochran, 1952) & $\mathrm{A} / \mathrm{G}$ \\
\hline & Trachycephalus venulosus (Laurenti, 1768) & $\mathrm{A} / \mathrm{G}$ \\
\hline \multirow[t]{6}{*}{ Leptodactylidae } & Leptodactylus fuscus (Schneider, 1799) & $\mathrm{A} / \mathrm{G}$ \\
\hline & Leptodactylus furnarius Sazima \& Bokermann, 1978 & $\mathrm{~A} / \mathrm{G}$ \\
\hline & Leptodactylus labyrinthicus (Spix, 1824) & $\mathrm{A} / \mathrm{G}$ \\
\hline & Leptodactylus mystacinus (Burmeister, 1861) & A \\
\hline & Leptodactylus cf. ocellatus (Linnaeus, 1758) & A \\
\hline & Leptodactylus podicipinus (Cope, 1862) & $\mathrm{A} / \mathrm{G}$ \\
\hline \multirow[t]{6}{*}{ Leiuperidae } & Eupemphix nattereri Steindachner, 1863 & $\mathrm{~A} / \mathrm{G}$ \\
\hline & Physalaemus cuvieri Fitzinger, 1826 & $\mathrm{~A} / \mathrm{G}$ \\
\hline & Physalaemus fuscomaculatus (Steindachner, 1864) & G \\
\hline & Pseudopaludicola aff falcipes & $\mathrm{A} / \mathrm{G}$ \\
\hline & Pseudopaludicola mystacalis (Cope, 1887) & $\mathrm{A} / \mathrm{G}$ \\
\hline & Pseudopaludicola sp. & $\mathrm{A} / \mathrm{G}$ \\
\hline \multirow[t]{2}{*}{ Microhylidae } & Elachistocleis sp. & $\mathrm{A} / \mathrm{G}$ \\
\hline & Dermatonotus muelleri (Boettger, 1885) & $\mathrm{A} / \mathrm{G}$ \\
\hline
\end{tabular}

